\title{
The stiff RhoAd from mevalonate to mutant p53
}

\author{
Giovanni Sorrentino $^{1} \cdot$ Fiamma Mantovani $^{2,3} \cdot$ Giannino Del Sal $^{2,3}$
}

Received: 2 February 2018 / Accepted: 6 February 2018 / Published online: 6 March 2018

(c) ADMC Associazione Differenziamento e Morte Cellulare 2018

Mutant p53 oncoproteins (mutp53), produced as result of missense mutations in the TP53 gene, actively promote tumour aggressive traits, metastatic dissemination and chemoresistance. Massive accumulation of mutp53 protein occurs in tumour cells [1] and is required for its oncogenic gain-of-function, indicating pharmacological destabilization of mutp53 as a potential anticancer therapeutic strategy [2]. In a recent issue of Nature Cell Biology [3], we reported that mechanical inputs, transduced by RhoA-dependent cytoskeletal tension, sustain mutp53 protein stability and that blocking this axis with mevalonate (MVA) pathway inhibitors curbs mutp53 accumulation in tumours (Fig. 1).

Relying on an extended protein interactome, which includes transcriptional regulators not bound by the wildtype counterpart, mutp53 drives tumour cell metabolic rewiring, migration/invasion, acquisition of stem traits and chemoresistance. In this context, mutp53 becomes constitutively stable, due to its engagement in complexes with the Hsp90 chaperone machinery, which prevents mutp53 poly-ubiquitination and proteasomal degradation [4]. Pharmacological destabilization of mutp53 by blocking Hsp90 with new generation inhibitors has proven effective to cause tumour regression in vivo [2]. Supporting the clinical efficacy of this strategy, HSP90 inhibitors were found to synergise with CCPT (concurrent cisplatin radiotherapy) in HNSCC cancers with mutant TP53 status [5]. This evidence further incites the quest for efficient and well-tolerated drugs targeting mutp53 stability as future chemotherapeutic treatments.

Giannino Del Sal

delsal@lncib.it

1 Laboratory of Metabolic Signaling, Institute of Bioengineering, Ecole Polytechnique Fédérale de Lausanne, Lausanne, Switzerland

2 Dipartimento di Scienze della Vita, Università degli Studi di Trieste, Trieste, Italy

3 Laboratorio Nazionale CIB, Area Science Park Padriciano, Trieste, Italy
Drug repositioning approaches represent a valid strategy to obtain hints on mechanisms sustaining oncogene activation, and to identify molecules able to interfere with these processes. Work by our group [3] and by others [6] highlighted that statins, a class of MVA pathway inhibitors and a very common drug used in the clinic for treatment of cardiovascular diseases, elicit mutp53 destabilization and reducing cancer cell proliferation. The MVA pathway is a conserved metabolic route that uses acetyl-CoA to produce cholesterol and other key biomolecules, some of which are required to support tumour development and progression. Specifically, the isoprenoid geranylgeranyl-pyrophosphate (GGPP) produced along the MVA pathway, is essential for post-translational modification and membrane anchoring of many proteins involved in aggressive cancer phenotypes. Among them, the small GTPase RhoA links ECM rigidity to intracellular actomyosin tension, acting as a mechanotransducer to drive tumour cell survival, proliferation and progression. In our work, we demonstrated that GGPP acts to stabilise the interaction of mutp53 with Hsp90, thus preventing its degradation (Fig. 1). We showed that this effect requires the histone deacetylase HDAC6, a direct activator of Hsp90 [7]. Interestingly, HDAC6 is controlled by changes in cytoskeleton dynamics [8], critical events in the crosstalk of transformed cells with the tumour microenvironment. Tumours display altered mechanotransduction compared to normal tissues, as cancer-associated fibrosis generates a dense and mechanically rigid extracellular matrix (ECM) leading to integrin clustering and activation in focal adhesions. These complexes induce RhoAdependent actin remodelling and actomyosin contractility (Fig. 1). It has become increasingly clear that mechanical cues presented to cells as a result of tissue stiffening, favour cancer development and progression. Interestingly, the levels of mutp53 appear frequently heterogenous within tumour tissues, with mutp53 over-expressing foci associated with fibrotic regions [9], suggesting that mutp53 stability may be locally influenced by tissue rigidity. 


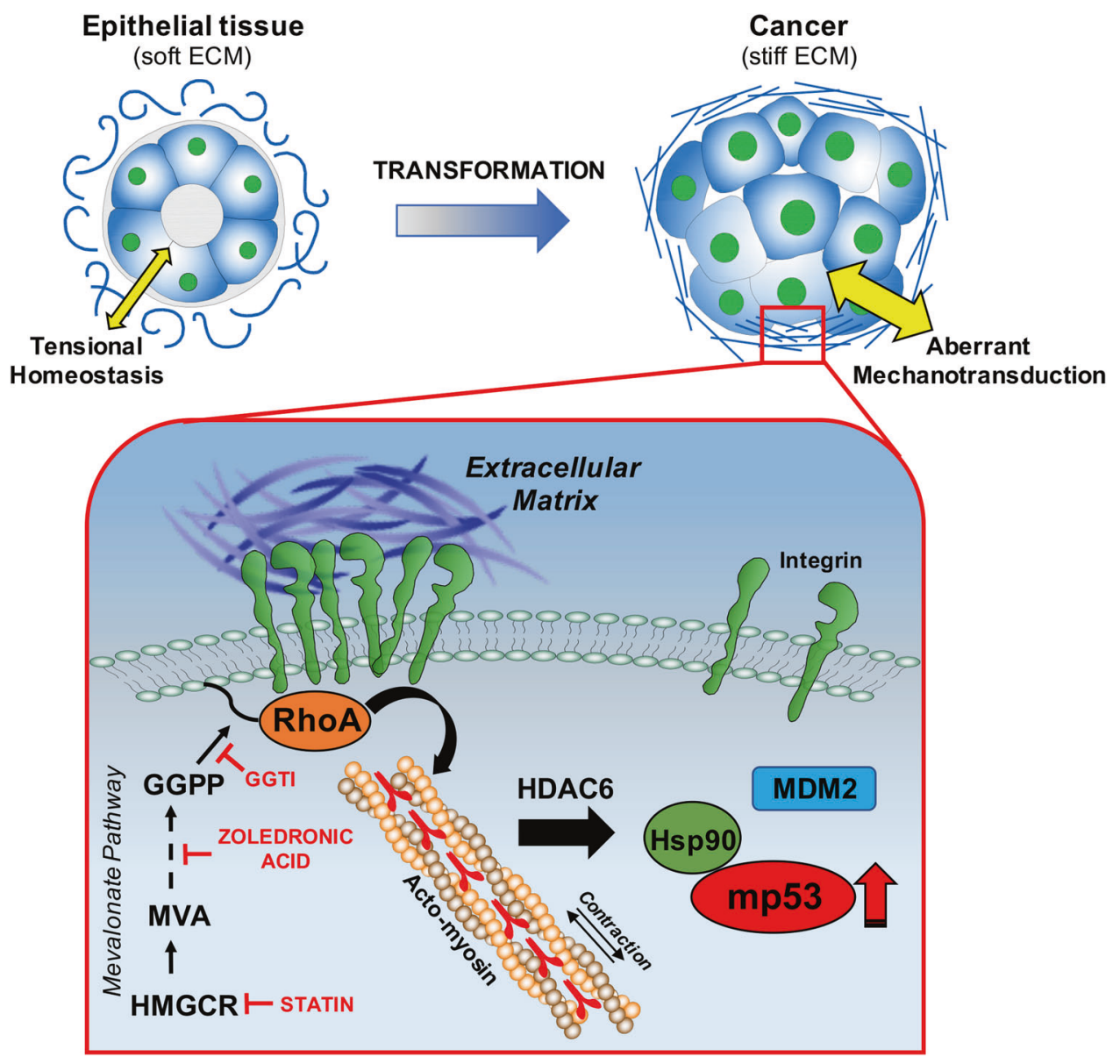

Fig. 1 Mutp53 accumulation in tumours is sustained by mechanical inputs through a mevalonate-RhoA-HDAC axis. Normal epithelial cells tune their contractility in response to tensional homoeostasis, but tumours exhibit altered mechanotransduction. During transformation, tumour-associated fibrosis generates a mechanically rigid matrix leading to integrin clustering and activation in focal adhesions. These complexes activate RhoA-dependent remodelling of filamentous actin and actomyosin contractility, thereby triggering Hsp90-mutp53 physical interaction and mutp53 stabilisation. This event requires HDAC6 and leads to functional inactivation of the MDM2 ubiquitin ligase. RhoA requires geranylgeranylation for proper membrane localisation

We reasoned that HDAC6 may promote mutp53 stabilisation downstream of mechanical inputs, transduced by RhoA-dependent cytoskeletal tension. In this context, GGPP produced by the MVA pathway appears crucial to sustain RhoA activation at the plasma membrane (Fig. 1). Consistently with this idea, we observed that inhibition of RhoA by different means (i.e., acting on its prenylation, enzymatic activity, or protein levels) caused mutp53 destabilization, similar to treatment with statins. To prove that ECM stiffness controls mutp53 stability, we grew non-tumorigenic mammary epithelial cells (MEC) from premalignant $\mathrm{p} 53^{\mathrm{R} 172 \mathrm{H} /}$ ${ }^{\mathrm{R} 172 \mathrm{H}}$ knock-in mice on substrates with varying rigidity. MEC displayed extremely low amounts of mutp53 when grown on soft hydrogels matching the elasticity of normal mammary and activation, using intracellular GGPP produced via the mevalonate (MVA) pathway, thus linking this metabolic pathway to transduction of mechanical signals and mutp53 stability. Inhibition of MVA flux using either statins or zoledronic acid, reduces intracellular levels of GGPP and consequently RhoA localisation and activation. Similar effects can be obtained blocking RhoA geranylgeranylation by treatment with inhibitors of GGTI protein geranylgeranyltransferase type I, such as GGTI-298. Therefore, administration of these treatments to cells growing on a rigid matrix in vitro, or to highly stiff tumours, can effectively interfere with cell mechanotransduction and thereby block mutp53 stabilisation and oncogenic activity

tissue. In striking contrast, culturing cells on a stiff substrate raised mutp53 abundance, proving that the physical properties of the local microenvironment can tune mutp53 levels. As expected, this effect required both HDAC6 and RhoA activity. Importantly, administration of MVA pathway inhibitors such as statin or zoledronic acid (ZA) was able to reduce the rigidity of cells grown on stiff matrix (as measured by atomic force microscopy) and to prevent mutp53 stabilisation, and it robustly decreased mechanosignalling and mutp53 accumulation in tumours (Fig. 1). These data represent the first evidence that inhibition of the Mevalonate/ RhoA axis can block the stroma-tumour mechanosignalling, thus representing a valuable approach to target its tumorigenic effects. 
Our findings provide several important implications for future investigation. Mutp53 has been previously shown to cooperate with SREBP in inducing the expression of MVA enzymes in tumour cells, thereby dismantling normal mammary tissue architecture [10]. Moreover the ability to promote integrin recycling implicates mutp53 in fostering cancer cell mechanoresponsiveness [11]. Notably, mutp53 expression is associated with increased activation of membrane-bound RhoA at the tumour invasive front and at cell poles [12, 13], and indeed it has been shown to induce the expression of the GEF-H1 oncogene, a RhoA exchange factor [14]. Now, identification of mutp53 as a downstream effector of aberrant MVA activation and tissue rigidity suggests that during tumour progression, TP53 mutation triggers positive feed-forward loops that further sustain mutp53 accumulation and oncogenic activities by rewiring metabolic and mechanoregulatory pathways. The intimate crosstalk of mutp53 with the mechanosensitive oncogenes YAP/TAZ, whose activation is sustained by MVA and actomyosin dynamics [15], is also enforced downstream of metabolic reprogramming and altered cell mechanics.

Given the uniquely high incidence of missense TP53 mutations across multiple tumour types, one can project that strategies aimed at blocking these networks would produce a huge impact on cancer treatment. Based on the notion that treatments leading to mutp53 destabilization have proven successful in pre-clinical and clinical settings, it can be expected that combination of statins with standard chemotherapy may improve treatment efficacy in mutp53expressing tumours, thus calling for urgent randomised clinical trials. Retrospective studies considering TP53 status could clarify the inconsistencies regarding the association of statin administration with good prognosis in different solid tumours, to allow rational implementation of statins for cancer precision medicine.

The emerging field of mechanomedicine focuses on targeting tissue mechanics for therapeutic purposes. In this respect, interfering with environmental mechanical cues and disrupting cell mechanoresponsiveness are considered promising strategies of anticancer therapeutic intervention. Our findings support the attractive opportunity to interfere with a metabolic pathway to alleviate the mechanical stress imposed by the rigidity of the tumour tissue. In this context, inhibitors of mevalonate flux (statins, ZA) or of RhoA geranylgeranylation (inhibitors of protein geranylgeranyltransferase type I) could effectively enhance therapeutic response, delay disease progression and improve patient survival acting on the circuit linking mechanical signals with mutp53 oncogenic activity.
Acknowledgements The work of Del Sal group is funded by the Italian Health Ministry (RF-2011-02346976), the Italian University and Research Ministry (PRIN-2015-8KZKE3), the Cariplo Foundation (grant no. 2014-0812), Associazione Italiana per la Ricerca sul Cancro (AIRC Special Program Molecular Clinical Oncology ' 5 per mille' grant no. 10016 and AIRC IG grant no. 17659) and BeneficentiaStiftung Foundation.

\section{Compliance with ethical standards}

Conflict of interest The authors declare that they have no conflict of interest.

\section{References}

1. Terzian $\mathrm{T}$, et al. The inherent instability of mutant p53 is alleviated by Mdm2 or p16INK4a loss. Genes Dev. 2008;22:1337-44.

2. Alexandrova EM, et al. Improving survival by exploiting tumour dependence on stabilized mutant p53 for treatment.. Nature. 2015;523:352-6.

3. Ingallina E, et al. Mechanical cues control mutant p53 stability through a mevalonate-RhoA axis. Nat Cell Biol. 2018;20:28-35.

4. Li D, et al. Functional inactivation of endogenous MDM2 and CHIP by HSP90 causes aberrant stabilization of mutant p53 in human cancer cells. Mol Cancer Res. 2011;9:577-88.

5. McLaughlin M, et al. HSP90 inhibition sensitizes head and neck cancer to platin-based chemoradiotherapy by modulation of the DNA damage response resulting in chromosomal fragmentation. BMC Cancer. 2017;17:86.

6. Parrales A. DNAJA1 controls the fate of misfolded mutant p53 through the mevalonate pathway. Nat Cell Biol. 2016;18:1233-1243.

7. Li D, et al. SAHA shows preferential cytotoxicity in mutant p53 cancer cells by destabilizing mutant p53 through inhibition of the HDAC6-Hsp90 chaperone axis. Cell Death Differ. 2011;18:1904-13.

8. Boyault C, et al. HDAC6, at the crossroads between cytoskeleton and cell signaling by acetylation and ubiquitination. Oncogene. 2007;26:5468-76.

9. Koga $\mathrm{T}$, et al. Heterogeneous distribution of P53 immunoreactivity in human lung adenocarcinoma correlates with MDM2 protein expression, rather than with P53 gene mutation. Int J Cancer. 2001;95:232-9.

10. Freed-Pastor WA, et al. Mutant p53 disrupts mammary tissue architecture via the mevalonate pathway. Cell. 2012;148:244-58.

11. Muller PA, et al. Mutant p53 drives invasion by promoting integrin recycling. Cell. 2009;139:1327-41.

12. Timpson $\mathrm{P}$, et al. Spatial regulation of RhoA activity during pancreatic cancer cell invasion driven by mutant p53. Cancer Res. $2011 ; 71: 747-57$.

13. Xia M, et al. Tumor suppressor p53 restricts Ras stimulation of RhoA and cancer cell motility. Nat Struct Mol Biol. 2007;14:215-23.

14. Mizuarai S, et al. Mutant p53 induces the GEF-H1 oncogene, a guanine nucleotide exchange factor-H1 for RhoA, resulting in accelerated cell proliferation in tumor cells. Cancer Res. 2006;66:6319-26.

15. Sorrentino G, et al. Metabolic control of YAP and TAZ by the mevalonate pathway. Nat Cell Biol. 2014;16:357-66. 